Una publicación de la Cátedra Libre Ciencia, Política y Sociedad.

Contribuciones a un pensamiento latinomericano

\section{Las mujeres y la ciencia: obstáculos y desafíos para lograr la equidad de género}

Resumen: A pesar de que las mujeres son mayoría entre los/las investigadores/as en nuestro país, están infra-representadas en las categorías superiores y en la toma de decisiones en las instituciones científicas y universitarias. Si bien en los últimos años se han tomado algunas medidas que facilitan la compatibilización de las tareas profesionales con la vida personal y familiar, todavía el sector científico y universitario no está preparado para facilitar el desarrollo académico de las mujeres. Sin embargo, éste no es el único escollo a sortear. Los estereotipos de género explican en gran medida los obstáculos que afrontan las mujeres para su permanencia y promoción. La visibilización de estas dificultades y la toma de conciencia de ellas por parte de las propias mujeres es, sin dudas, el inicio del cambio. Se presenta un panorama de esta situación y se proponen algunas medidas para promover la igualdad de oportunidades entre mujeres y hombres, que deberían ser una prioridad para las instituciones del sector.

Palabras clave: Mujeres en ciencia y tecnología, científicas, estereotipos de género en CyT, ciencia y género, desigualdades en CyT.

Women and science: obstacles and challenges to achieve gender equity

Abstract: Although women are the majority among researchers in our country, they are underrepresented in the higher categories and in decision-making positions in scientific institutions and universities. Although in recent years some measures have been taken to facilitate the compatibility between professional and personal/family life, the scientific and university sector is still not prepared to support the academic development of women. However, this is not the only obstacle to overcome, gender stereotypes largely explain the barriers womenface for their permanenceand promotion. The visibility of these difficulties and the awareness of them by women themselves is, without doubt, the beginning of change. An overview of this situation is presented and some measures are proposed to promote equal opportunitiesbetweenwomenandmen, whichshouldbeapriorityfortheinstitutionsofthesector Key words: Women in science and technology, scientists, gender stereotypes in S\&T, science and gender, inequalities in S\&T.

Mulheres e ciência: obstáculos e desafios para alcançar a equidade de gênero

Resumo: Embora as mulheres sejam a maioria entre os pesquisadores em nosso país, elas estão sub-representadas nas categorias mais altas e na tomada de decisões em instituições científicas e universitárias. Embora nos últimos anos tenham sido adotadas algumas medidas que facilitam a compatibilidade das tarefas profissionais com a vida pessoal e familiar, o setor científico e universitário ainda não está preparado para facilitar o desenvolvimento acadêmico das mulheres. No entanto, esse não é o único obstáculo a ser superado. Os estereótipos de gênero explicam amplamente os obstáculos que as mulheres enfrentam para sua permanência e promoção. A visibilidade dessas dificuldades e a conscientização delas pelas próprias mulheres são, sem dúvida, o começo da mudança. É apresentada uma visão geral dessa situação e são propostas algumas medidas para promover a igualdade de oportunidades entre homens e mulheres, o que deve ser uma prioridade para as instituições do setor. Palavras chave: Mulheres em ciência e tecnologia, cientistas, estereótipos de gênero em C\&T, ciência e gênero, desigualdades em C\&T.

\section{Ciencia Tecnología y Política \\ Año 2 Nº 3 jul- dic 2019}

a

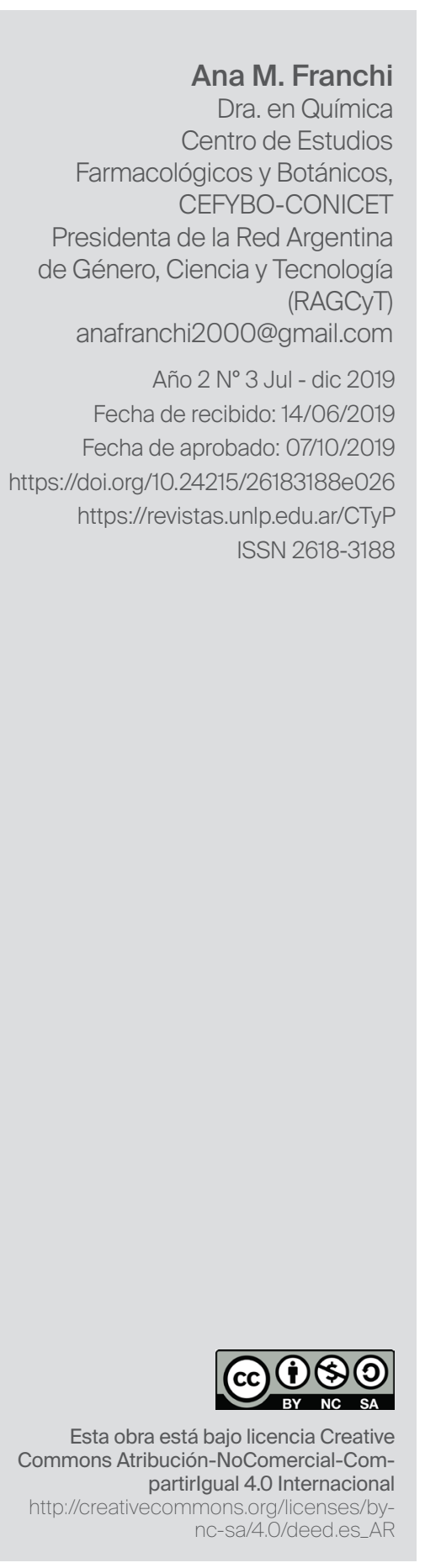

UNIVERSIDAD

NACIONAL

DE LA PLATA 
| Ciencia, Tecnología y Política | Año 2 | №3 | Julio-Diciembre 2019 | ISSN 2618-3188 | www.revistas.un|p.edu.ar/CTyP |

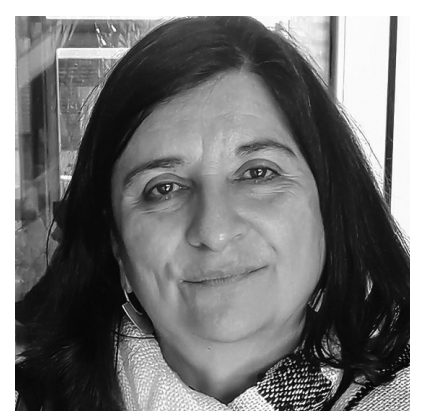

\section{Ana M. Franchi}

Dra. en Química

Centro de Estudios Farmacológicos

y Botánicos, CEFYBO-CONICET

Presidenta de la Red Argentina

de Género, Ciencia y Tecnología

(RAGCyT)

anafranchi2000@gmail.com

\section{Las mujeres y la ciencia: obstáculos y desafíos para lograr la equidad de género}

Resumen: A pesar de que las mujeres son mayoría entre los/las investigadores/as en nuestro país, están infra-representadas en las categorías superiores y en la toma de decisiones en las instituciones científicas y universitarias. Si bien en los últimos años se han tomado algunas medidas que facilitan la compatibilización de las tareas profesionales con la vida personal y familiar, todavía el sector científico y universitario no está preparado para facilitar el desarrollo académico de las mujeres. Sin embargo, éste no es el único escolloa sortear. Los estereotipos de género explican en gran medida los obstáculos que afrontan las mujeres para su permanencia y promoción. La visibilización de estas dificultades y la toma de conciencia de ellas por parte de las propias mujeres es, sin dudas, el inicio del cambio. Se presenta un panorama de esta situación y se proponen algunas medidas para promover la igualdad de oportunidades entre mujeres y hombres, que deberían ser una prioridad para las instituciones del sector.

\section{Mujeres, ciencia e historia}

La relación de las mujeres con la ciencia no es ni ha sido fácil. La historia de esta relación está determinada por procesos históricos y sociales y por diversas instituciones que se han entronizado en lugares del "saber", tanto religiosas como académicas.

En la actualidad son muchas las mujeres que se dedican a la ciencia, aunque para ello deben franquear numerosas barreras de tipo social y laboral (compaginar la vida familiar, enfrentar prejuicios culturales, poco reconocimiento profesional, desigualdad salarial, etc.). A pesar de ser un colectivo numeroso, son muy pocas las mujeres que acceden a los puestos más altos en los organismos de ciencia y técnica o a las cátedras universitarias. Todavía existe el llamado "techo de cristal" que bloquea la presencia de mujeres en los tramos finales de la escala profesional.

A modo de ejemplo, de los premios nobel en ciencia (Fisica, Quimica y Medicina) instituidos desde 1901, 616 han sido otorgados a hombres y solo 19 a mujeres, es decir una mujer cada 32 hombres. 
En términos generales, cuando se habla de las mujeres científicas, son escasos los nombres que se mencionan, con casi la única excepción de Marie Curie. Es frecuente aludir a la ausencia de mujeres en el desarrollo científico, nada más alejado de la realidad. Sin embargo, son muchas las mujeres que se han destacado en la ciencia a lo largo de la historia y demasiadas las que fueron discriminadas, maltratadas e incluso asesinadas por pretender acercarse al "saber científico".

Para ellas no fue fácil. Hypatia (Siglo I), filósofa y matemática, se interesó también por la tecnología y diseñó diversos instrumentos tales como el astrolabio plano que se usaba para medir la posición de las estrellas y planetas (Alic, 2005). La mayoría de sus escritos formaron libros de texto para sus estudiantes. Llegó a simbolizar el conocimiento y la ciencia que los primeros cristianos identificaron con el paganismo. Eran tiempos difíciles para los paganos, porque el cristianismo se estaba imponiendo en Alejandría (que en aquellos tiempos estaba bajo el dominio romano). Hypatia se negó a traicionar sus ideas y convertirse al cristianismo, por lo que fue acusada de conspiración contra el líder cristiano de Alejandría. Fue asesinada brutalmente mientras regresaba a su casa en un carruaje, la golpearon y arrastraron por toda la ciudad.

Durante la Edad Media, para las mujeres que quisieron acceder a la educación, su única salida fue la vida monástica y conventual, donde pudieron estudiar, aprender e incluso llegar a ser auténticas eruditas. Para muestra tenemos a Hildegarda de Bingen (1098-1179), quien fue la primera en afirmar que el sol era el centro del sistema planetario, además de escribir numerosas obras de medicina y farmacia (Caso, 2006).

Las tan desacreditadas brujas fueron en su gran mayoría, mujeres con conocimientos específicos en alquimia, que usaron para elaborar recetas de perfu- mería y cosmética, desarrollar técnicas de destilación, extracción y sublimación. Estas mujeres eran parteras, alquimistas, perfumistas, nodrizas y cocineras, que tenían conocimiento en campos como la anatomía, la botánica, la sexualidad, la reproducción y que prestaban un importante servicio a la comunidad. Sin embargo, su tarea no sólo no era apreciada por el poder dominante sino que fueron temidas y perseguidas. De los cientos de miles de víctimas de la Inquisición, las mujeres superaron en número a los hombres, en algunos lugares incluso en una proporción de diez a uno (Blázquez Graf, 2008)

La creación de las universidades durante el siglo XIII no mejoró la situación. En 1220, la Facultad de Medicina de la Universidad de París exigió que sólo los hombres solteros pudiesen ejercer la medicina.

El acceso a las universidades de Oxford y Cambridge estaba totalmente cerrado a las mujeres, por lo que ellas podían adquirir únicamente conocimientos prácticos. Recién en 1947, la Universidad de Cambridge permite a las mujeres obtener títulos completos (Roach, 1959).

Mucho antes, en 1883, Cecilia Grierson ingresa a la Facultad de Medicina de la Universidad de Buenos Aires y se convierte, pocos años después, en la primera médica argentina (Binda, Silveira y Krämer, 2010).

Las mujeres ganaron sólo uno de cada veinte Premios Nobel. La primera fue Marie Sklodowska Curie, quien recibió en 1903 el Premio Nobel de Física compartido con su marido, y en 1911 el Premio Nobel de Química (Nobel Media AB, 2019). De hecho, su hija, Irène Joliot-Curie, que también recibió el premio Nobel de Química, es habitualmente ignorada.

A pesar de que la ciencia, la tecnología y la innovación son motores clave del crecimiento económico, las mujeres siguen representando una minoría de 
los investigadores del mundo, como hace muchos siglos.

Para el año 2015, los promedios regionales para la participación de las investigadoras indican que las mujeres representan el 28,8\% de los investigadores del mundo: el 32,3\% en Norteamérica y Europa Occidental y el 45,4\% en América Latina y el Caribe (UNESCO, 2018).

\section{Las mujeres en el CONICET y las Universida- des Nacionales}

El Consejo Nacional de Investigaciones Cientificas y Técnicas (CONICET) tiene un sistema de becas y una carrera de investigador. La carrera de investigador tiene cinco jerarquías: asistente, adjunto, independiente, principal y superior. Desde el año 2007, las mujeres investigadoras superan en número a sus colegas varones y actualmente representan el $53,5 \%$ del total de los investigadores del organismo (CONICET, s/f). Sin embargo, cuando se analiza la distribución en las distintas categorías de la carrera del investigador, las mujeres siguen siendo mayoría sólo en las categorías más bajas de la carrera. La creciente presencia de las mujeres registrado (figura 1) en el total de investigadores/as muestra importantes diferencias en la composición por género según las categorías de la Carrera de Investigador/a Científico/a -CIC-: mientras que las mujeres constituyen una clara mayoría en las etapas de Asistentes y Adjuntos/as, su presencia disminuye sistemáticamente en la medida que se progresa en la carrera, hasta el punto de que constituyen menos de la cuarta parte de los investigadoras/es Superiores (23\%)

Si bien en los últimos años se han tomado algunas decisiones que favorecen la permanencia y el desarrollo de las mujeres (como la licencia de maternidad para becarias, la prolongación de la beca por el tiempo de la licencia, y otras), todavía el sector científico no está preparado para facilitar el desarrollo académico de las mujeres.

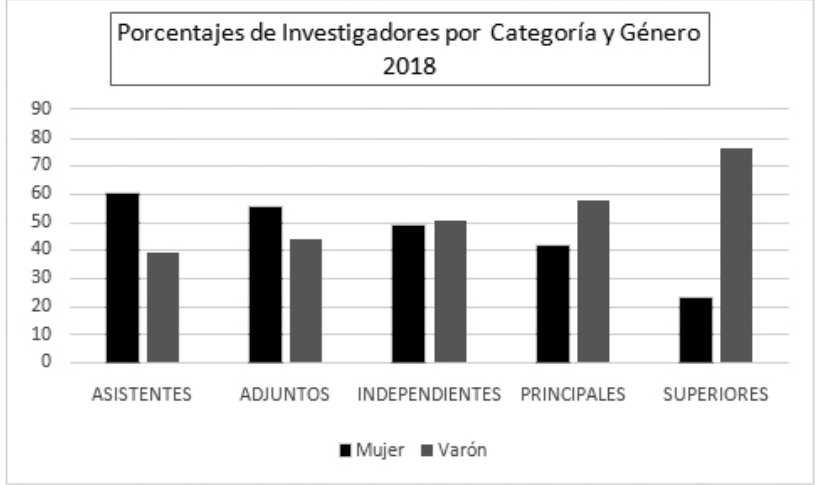

Figura 1: Porcentajes de Investigadores por Categoría y Género. Fuente: Base de Datos del CONICET. Gerencia de Recursos Humanos. Oficina de Información Estratégica en RRHH

El ingreso a la Carrera del investigador $(\mathrm{CIC})$ y las primeras etapas en la carrera coinciden con el período de vida reproductiva de las mujeres, lo que podría generar obstáculos para conciliar sus desarrollos académicos con su vida familiar. Estos obstáculos son semejantes a los que afrontan las trabajadoras en todos los sectores, en particular durante los primeros meses de vida de sus hijos/as. La brevedad de la duración de las licencias por maternidad y las dificultades en el acceso a servicios de cuidado generan el interrogante acerca del modo en que las investigadoras del CONICET afrontan estos obstáculos; de hecho es frecuente que algunas investigadoras opten por postergar la maternidad en función de promocionar en la carrera científica. Considerando esta situación, el CONICET adoptó medidas que tienden a la equidad de género, pero esto parece no impactar aun suficientemente en la promoción de las mujeres en la carrera del investigador.

La participación de las mujeres en posiciones de decisión es aún menor. Los centros e institutos del CONICET, denominados Unidades Ejecutoras, son unidades de investigación y servicios que, bajo la responsabilidad de un director, realizan tareas de investigación científica, tecnológica o de desarrollo. El Director es el responsable institucional de la unidad y tiene a su cargo la responsabilidad de la administra- 
ción de los recursos humanos y económicos o patrimoniales. Actualmente hay más de 260 Unidades Ejecutoras y sólo el $25 \%$ es dirigido por mujeres.

Los Centros Científico Tecnológicos (CCT) del CONICET son estructuras funcionales de amplio espectro temático, cuyo objetivo primordial es asegurar un ámbito apropiado para la ejecución de investigaciones científicas, tecnológicas y de desarrollo en el espacio físico y de influencia que le compete. Actualmente el CONICET cuenta con dieciséis CCTs, sólo tres de ellos dirigidos por mujeres.

El Directorio del CONICET es la autoridad de aplicación e interpretación de las disposiciones del Estatuto que rige el organismo. El Decreto 1661/96, aún vigente, establece que el CONICET es conducido por un Directorio integrado por 8 miembros y un presidente, designados por el Poder Ejecutivo Nacional; actualmente sólo una mujer participa en el directorio. (ver figura 2)

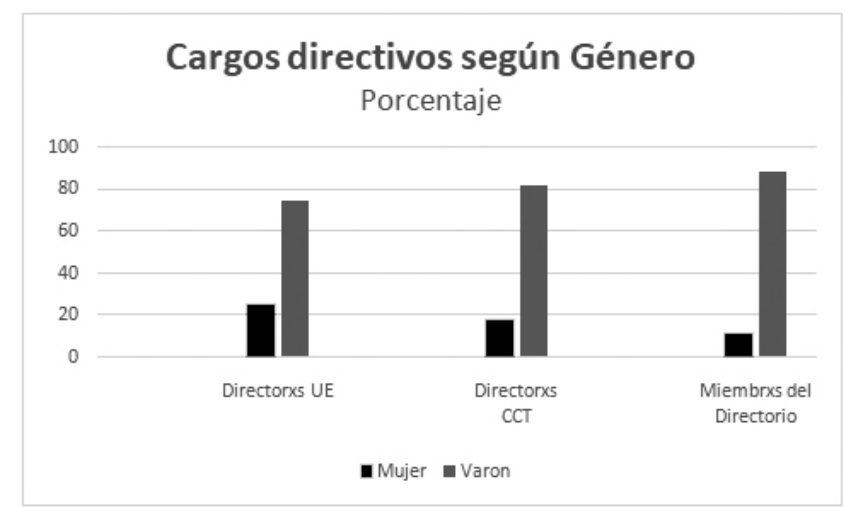

Figura 2: Porcentajes de cargos directivos según Género. Fuente: Base de Datos del CONICET. Gerencia de Recursos Humanos. Oficina de Información Estratégica en RRHH

Algo similar ocurre en las universidades nacionales. Según datos de 2018 de la Secretaría de Políticas Universitarias, a pesar de que las mujeres representan casi el 60\% de las y los ingresantes y más del $60 \%$ de las y los graduados/as, sólo el 11\% de los/as rectores/as son mujeres. Ver figura 3

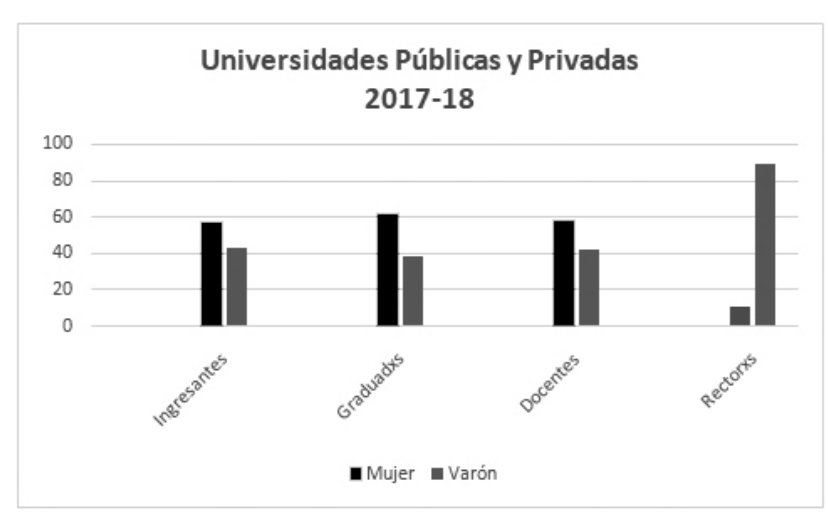

Figura 3: Porcentaje de mujeres y varones. Fuente: Departamento de Información Universitaria - DNPeIU - SPU

\section{Obstáculos para lograr la equidad de género}

Ahora bien, ¿por qué sucede esto? Las posibles explicaciones son muchas, pero una de ellas, sin dudas, son los estereotipos de género. El mayor estudio sobre el estereotipo de género en la ciencia, realizado sobre más de 350.000 participantes de ambos sexos en 66 países, reveló que el 83\% asocia la actividad científica con los hombres más que con las mujeres. Este trabajo publicado en el Journal of Educational Psychology, mostró que la Argentina también figura entre los países más prejuiciosos en este terreno (Miller, Eagly \& Linn, 2015).

Una encuesta europea encargada por la Fundación L'Oréal en la que participaron 5000 personas, señala que seis de cada 10 personas creen que las mujeres no sirven para ser científicas de alto nivel. Las "razones" que alegan son tan variopintas como que a las mujeres les falta interés por la ciencia, perseverancia, espíritu racional, sentido práctico y espíritu analítico, entre otras.

Los estereotipos de género se ven claramente expuestos en el llamado "efecto Jennifer y John" basado en un experimento realizado por la Universidad de Yale en el año 2012, en el que se pedía a docentes calificados de los departamentos de ciencias (física, química y biología) de seis universidades norteamericanas, que valoraran la solicitud presen- 
tada por un estudiante (John) o una estudiante (Jennifer) ficticios, para ocupar, en sus departamentos, el cargo de responsable del laboratorio (lab manager). Una de las peculiaridades del experimento fue que la documentación que evaluaban era la misma y sólo se diferenciaba en el nombre y sexo de la persona solicitante (Moss-Racusin et al. 2012). A cada responsable se le pidió la valoración de la documentación enviada en función de tres aspectos (en una escala de 1 a 7): competencia (competence), contratabilidad (hireability) y nivel de tutorización (mentoring); también se les pidió que indicaran qué remuneración consideraban que se tenía que asignar al postulante en cuestión, en función de sus méritos. Jennifer fue considerada menos competente y menos contratable que John, y el/la responsable estaba dispuesto a dedicarle menos tiempo de tutoría. En el aspecto relativo al salario, estaba dispuesto/a a pagar 26.508 USD a Jennifer, mientras que a John le hubieran pagado 30. 238 USD. Lo preocupante es que estos estereotipos no sólo persisten aún, sino que aparecen ya desde el inicio de la educación de las niñas y los niños.

En los años ochenta una investigación realizada en América del Norte pidió a casi 5.000 niños y niñas estadounidenses y canadienses que dibujaran un científico, sólo 28 niños $(0,6 \%)$ representaba a una mujer científica (Chambers, 1983). La asociación de la ciencia con los varones se ha debilitado, pero aún persiste; en un estudio más reciente (Farland-Smith, 2009), sólo el 35\% de los niños estadounidenses, ante el mismo pedido, representaba a una mujer científica.

En una investigación realizada por la Cátedra Regional UNESCO Mujer, Ciencia y Tecnología en América Latina - FLACSO Argentina con la Asociación Civil Chicos.net, y con el apoyo de Disney Latinoamérica, en tres ciudades latinoamericanas (Buenos Aires, Ciudad de México y San Pablo), se evaluó cómo se vinculan niños y niñas en los primeros años de escolaridad con las áreas y actividades de Ciencia y Tecnología para detectar si en este proceso inciden estereotipos y sesgos de género (Bonder, 2017). En la ciudad de Buenos Aires, uno de cada dos padres/ madres creen que los varones tienen más habilidad para el uso de la tecnología y que los niños tienen mejor rendimiento que las niñas en Tecnología e Informática, aunque niños y niñas se entretienen con ella por igual.

Desde la visión de los/as docentes, los varones aventajan en rendimiento a las chicas en Informática y explican que estas diferencias se deben a que ellos poseen más capacidades para una de estas áreas.

Asimismo, un estudio del año 2009 demostró que cuanto más baja es la igualdad de género en una nación, mayor es la brecha de aptitud matemática entre niños y niñas, lo que sugiere que la cultura, no la biología, es la culpable (Hyde \& Mertz, 2009).

Otra desigualdad que afecta a la promoción de las mujeres en ciencia y tecnología es el financiamiento de sus investigaciones. Por ejemplo, menos mujeres que hombres se presentan al prestigioso programa del European Research Council y las propuestas presentadas por mujeres tienen una probabilidad algo menor de resultar elegidas. En los últimos diez años, el porcentaje de proyectos presentados por mujeres ha aumentado ligeramente, pero el de proyectos otorgados a mujeres no ha cambiado en forma significativa. El sesgo se amplía a medida que los proyectos aumentan en monto y en 2016, sólo el 19\% de los Advanced Grants, las más cuantiosas, fueron destinadas a mujeres. Un trabajo realizado por la Secretaría de Gobierno de Ciencia, Tecnología e Innovación productiva demuestra (ver figura 4) que en la Argentina, en todas las áreas de conocimiento estudiadas, las mujeres reciben menos financiación en los subsidios más importantes de 
Mediana de montos de proyectos financiados según gran área del conocimiento

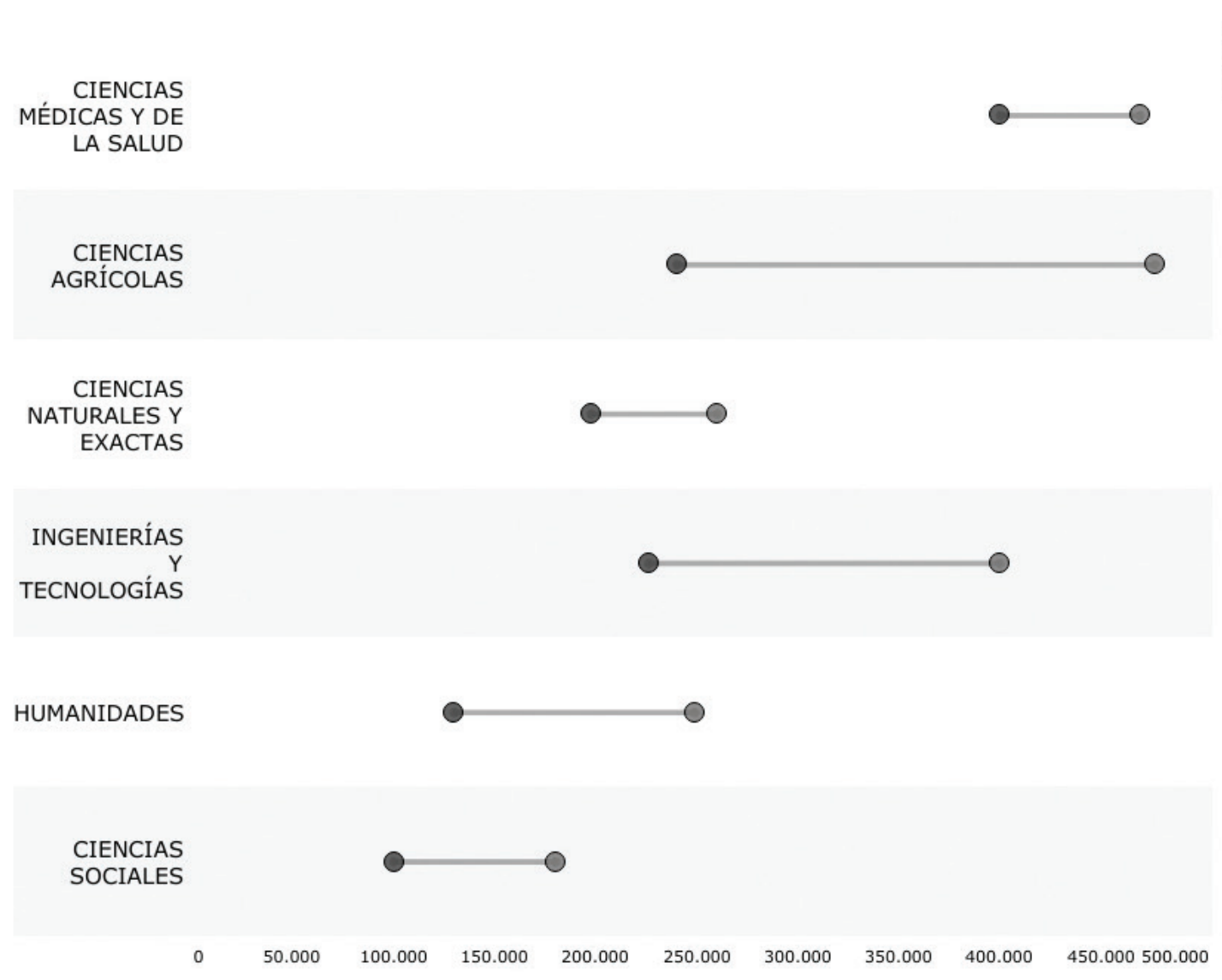

Seleccione una entidad financiadora

CONICET

PONCYT

INTA

Seleccione para resaltar

$\square$ Mujeres

$\square$ Varones

Figura 4: Monto de financiamiento de los subsidios FONCYT por sexo y disciplina. Fuente: SICYTAR.

nuestro país, que son otorgados por el Fondo para la Investigación Científica y Tecnológica (FONCyT). Dentro de sus propios colegas, el apoyo hacia las postulantes mujeres es desigual: a nivel mundial, las mujeres tienen alrededor de un 10\% menos de probabilidades que los varones de recibir calificaciones "excelentes" en las cartas de recomendación para puestos postdoctorales en Ciencias de la Tierra. El hallazgo se mantiene independientemente del género de quien recomiende o en qué parte del mundo trabaja el solicitante (Dutt, et al., 2016). Estas cartas son muy importantes para acceder a puestos de doctorado, post-doctorado, becas y al primer empleo como profesor/a.

Otro factor que contribuye a dificultar la carrera de las mujeres en ciencia es la discriminación en la eva- luación por parte de alumnos. Las calificaciones de los estudiantes juegan un papel importante en los resultados profesionales de los docentes de Educación Superior. Un estudio realizado en la Universidad de North Alabama (MacNell, Driscoll \& Hunt, 2015) mostró que los estudiantes calificaron la identidad masculina significativamente más alta que la identidad femenina, independientemente del género real del docente que pudo ser escondido al realizar este estudio con clases virtuales; de esta forma, los docentes operaban bajo dos identidades de género diferentes. La menor calificación hacia las mujeres, dado el papel vital que desempeñan las calificaciones de los estudiantes en las trayectorias profesionales académicas, muestra nuevamente sesgos de género que entorpecen las carreras de las mujeres. 


\section{Recomendaciones y propuestas}

Se han mencionado sólo algunas de las vallas que afrontan las mujeres en el pedregoso camino que es para ellas una carrera científica. La visibilización de estas dificultades y la toma de conciencia de ellas por parte de las propias mujeres es, sin dudas, el inicio del cambio.

En el año 2015, la Organización de Naciones Unidas (ONU, 2016) proclamó que todos los 11 de febrero sean conmemorados como el "Día Internacional de la Mujer y la Niña en la Ciencia" con el fin de lograr el acceso y la participación plena y equitativa en la ciencia para las mujeres y las niñas, y además, para lograr la igualdad de género y el empoderamiento de las mujeres y las niñas. Dado el papel central que ocupan la ciencia, la tecnología y la innovación como ejes esenciales para un mejor desarrollo socio-económico, es de suma importancia que las mujeres participen plenamente y de manera equitativa en estas actividades.

Como ya se mencionó, las instituciones de Ciencia y Tecnología y las Universidades, incluso los departamentos o centros aparentemente progresistas y equitativos, siguen siendo profundamente desiguales e inequitativos para las mujeres investigadoras. La incorporación de medidas para promover la igualdad de oportunidades entre mujeres y hombres debe ser una prioridad para las instituciones del sector científico tecnológico.

El desarrollo profesional de las mujeres en ciencia y tecnología puede beneficiarse con políticas de educación para promover las disciplinas y áreas donde las mujeres están menos presentes como las ingenierías, las matemáticas, o las vinculadas con la tecnología. Es imprescindible el desarrollo de programas de apoyo a mujeres científicas, premios y políticas de igualdad de género en los sistemas nacionales de investigación, y de facilidades para el cuidado de los/as hijos/as.

Por otro lado, conocer la existencia de mujeres científicas, su trabajo y las circunstancias en que lo desarrollaron o lo desarrollan puede ser inspirador de vocaciones en niñas y jóvenes.

Es mucho lo que falta para que mujeres y varones tengan iguales oportunidades en sus carreras, Io que no resultará posible sin cambios que garanticen que las voces, las contribuciones y la excelencia de las científicas asciendan a la cima de las agendas políticas, de la investigación y de la sociedad civil.

\section{Bibliografía:}

Alic, M. (2005). El legado de Hipatia. México: Siglo XXI.

Binda, M., Silveira, R. y Krämer, C. (2010). Cecilia Grierson, la primera médica argentina. Revista Argentina de Radiología [en linea]. 74(4), pp. 361365.

Blázquez Graf, N. (2008). El retorno de las brujas. Incorporación, aportaciones y críticas de las mujeres a la ciencia. Universidad Nacional Autónoma de México, Centro de Investigaciones Interdisciplinarias en Ciencias y Humanidades.

Bonder, G. (2017) Infancia, Ciencia y Tecnología: un análisis de género desde el entorno familiar, educativo y cultural. Cátedra Regional UNESCO Mujer Ciencia y Tecnología en América Latina - FLACSO Argentina con la Asociación Civil Chicos.net, y con el apoyo de Disney Latinoamérica. Recuperado de http://www.codajic.org/sites/www.codajic.org/ files/Infancia,\%20Ciencia\%20y\%20tecnolog\% C3\%ADa.pdf

Caso, A. (2006). Las olvidadas. Una historia de mujeres creadoras. Barcelona: Círculo de Lectores.

Chambers, D. W. (1983). Stereotypic images of 
the scientist: The drawa-scientist test. Science Education, 67, 255-265. doi:10.1002/sce .3730670213

CONICET (s/f). Base de Datos. Gerencia de Recursos Humanos. Oficina de Información Estratégica en RRHH.

Dutt, K; Pfaff, D.; Bernstein, A.; Dillard, J. \& Block, C. (2016). Gender differences in recommendation letters for postdoctoral fellowships in geoscience. Nature Geoscience, 9, 805-808. http://dx.doi.org/10.1038/ngeo2819

Farland-Smith, D. (2009). How does culture shape students' perceptions of scientists? Cross-national comparative study of American and Chinese elementary students. Journal of Elementary Science Education, 21, 23- 42.

Hyde, J. \& Mertz, J. (2009) Gender, culture, and mathematics performance. PNAS106 (22), 88018807. https://doi.org/10.1073/pnas.0901265106

MacNell, L., Driscoll, A. \& Hunt, A.N. (2015). What's in a Name: Exposing Gender Bias in Student Ratings of Teaching. Innovative Higher Education 40 (4), 291-303. https://doi.org/10.1007/ s10755-014-9313-4

Miller, D. I., Eagly, A. H. \& Linn, M. C. (2015). Women's representation in science predicts national gender-science stereotypes: Evidence from 66 nations. Journal of Educational Psychology, 107(3), 631-644.

Moss-Racusin, C.; Dovidio, J.; Brescoll, V.; Graham, M. \& Handelsman, J. (2012). «Science faculty's subtle gender biases favor male students.». PNAS 109 (41): 16474-16479.

Nobel Media AB (23 de septiembre de 2019). Nobel Prize awarded women. NobelPrize.org. Recuperado de https://www.nobelprize.org/pri zes/lists/nobel-prize-awarded-women/
Organización de Naciones Unidas (ONU) (2016). Resolución aprobada por la Asamblea General el 22de diciembre de 2015 70/212. Día Internacional de la Mujer y la Niña en la Ciencia. Recuperado de https://www.un.org/en/ga/search/view_doc.as p?symbol=A/RES/70/212\&Lang $=S$

Roach, J.P.C. (1959). 'The University of Cambridge: Epilogue (1939-56)', in A History of the County of Cambridge and the Isle of Ely: Volume 3, the City and University of Cambridge (pp. 307-312). London: Victoria County History

UNESCO (2018). Institute for Statistics, Junio 2018. Ministerio de Educación, Cultura, Ciencia y Tecnología (s/f). Ciencia, Tecnología e Innovación Productiva. Equidad de Género en Ciencia, Tecnología e Innovación. Estadísticas de Género en Ciencia, Tecnología e Innovación. Medianas de montos financiados. Recuperado de https://www. argentina.gob.ar/ciencia/sact/medianas-de-mo ntos-financiados 